\title{
Clomiphene citrate rescue of spermatogenesis in men with infertility while remaining on finasteride: A case report
}

\author{
Christopher Wu, MD; Ellen Forbes, $M D_{;}^{2}$ Keith Allen Jarvi, $M D^{2}$
}

'Division of Urology, Department of Surgery, McMaster University, Hamilton, ON, Canada; '2Division of Urology, Department of Surgery, Mount Sinai Hospital, University of Toronto, Toronto, ON, Canada

Cite as: Can Urol Assoc J 2017;11 (3-4):E122-3. http://dx.doi.org/10.5489/cuaj.4191 Published online March 16, 2017

\section{Introduction}

Finasteride is a selective inhibitor of type-2 5 -alpha reductase enzyme, preventing the conversion of testosterone to dihydrotestosterone (DHT). ${ }^{1}$ Finasteride is used as an oral drug for the treatment of benign prostatic hyperplasia at the $5 \mathrm{mg} /$ day dose and is also used for the treatment of androgenic alopecia, or male pattern hair loss, at a dosage of 1 mg orally. ${ }^{2}$ There are now a number of studies documenting that finasteride, even at the low dose of $1 \mathrm{mg}$, may have an adverse effect on fertility for some men. ${ }^{3-6}$ In the largest study, Samplaski et al reported that sperm counts improved over 11 -fold once the men had stopped the finasteride, but most of the men did not recover to normal sperm parameters. ${ }^{3}$

Presently, if men using finasteride are subfertile, the recommendation is to stop the finasteride and wait for a recovery of spermatogenesis. ${ }^{3}$ There has been no report of the use of any hormone therapy, such as clomiphene citrate, gonadotropin, or aromatase inhibitors, to treat subfertile men on finasteride. This is the first case report of the use of clomiphene citrate to improve fertilty for a man on finasteride.

\section{Case report}

A 38-year old male with azoospermia was referred for infertility evaluation. He presented with a one-year history of secondary infertility. He had been taking finasteride $1 \mathrm{mg} /$ day for two years to treat androgenic alopecia and had used oral testosterone for three months (stopping six months before the semen testing showing azoospermia). Initial investigations at the time of his first visit showed a low total morning testosterone of $4.04 \mathrm{nmol} / \mathrm{L}$, but normal follicle-stimulating hormone (FSH), luteinizing hormone (LH), estradiol, and prolactin levels.

He complained of symptoms of hypo-androgenism and was subsequently started on clomiphene citrate (CC) 25 mg orally every other day, but at the patient's request he remained on finasteride. At six months, his semen parameters improved to a count of $8.9 \times 10^{6} / \mathrm{ml}, 20 \%$ motility, and $5 \%$ normal forms, and his serum testosterone increased to 16 $\mathrm{nmol} / \mathrm{L}$. He and his partner are now attempting to conceive with intrauterine insemination.

\section{Discussion}

Finasteride use, even at the low dose level of $1 \mathrm{mg}$ /day used for androgenic alopecia, appears to cause infertility for a small fraction of men. By blocking the conversion of testosterone to dihydro-testosterone (the more potent androgen), finasteride reduces the overall "androgenicity" in the tissues. This effect is used to treat androgenic alopecia and prostate hypertrophy. It is postulated that the fraction of men who have infertility while taking finasteride are more sensitive to the reduced "androgenicity" caused by finasteride in the seminiferous tubules than other men. ${ }^{3}$ Amory et al, in a large series, did not demonstrate an overall negative impact of finasteride on spermatogenesis in men with normal fertility. ${ }^{2}$ However, this group did report that $5 \%$ of the subjects taking finasteride in their study experienced a profound reduction in their total sperm counts to less than $10 \%$ of baseline values. There is mounting evidence to suggest that certain individuals may be more sensitive to the fertility effects of $5 \alpha$-reductase inhibition and that finasteride use leads to infertility in some men..$^{3-6}$ The largest published series has shown that for men with infertility who are taking finasteride, sperm counts increased 11 -fold once the finasteride was discontinued. ${ }^{3}$

While Samplaski et al recommended that infertile men discontinue finasteride use, many of these men will be unwilling to take this advice. ${ }^{3}$ Faced with this clinical situation, what therapies could we offer these men to improve semen parameters?

$\mathrm{CC}$ is widely used as empiric therapy to treat men with low sperm counts. ${ }^{7}$ Through blockade of the estrogen receptor at both the level of the hypothalamus and pituitary, estrogenic negative feedback inhibition of FSH and $\mathrm{LH}$ secretion 
is prevented. The increased concentrations of FSH and $\mathrm{LH}$ lead to increased testosterone production (higher circulating and intratesticular testosterone levels)., ${ }^{3,7}$ The efficacy of CC therapy on fertility is controversial, with studies showing improvement in semen parameters, but no statistically significant improvement in pregnancy rates. ${ }^{8,9}$

For our patient, CC therapy resulted in increases in sperm count. In theory, this improved sperm count could have been due to the CC alone (since we don't have semen parameters prior to the initiation of the finasteride, the azoospermia could have been a pre-existing condition) or could have been a result of the CC therapy increasing the intratesticular androgen levels to compensate for the reduced "androgenicity" related to the finasteride use. It is also possible, but unlikely, that the oral testosterone taken for three months, but stopped six months prior to the semen testing, caused the azoospermia. In a contraceptive study, oral testosterone undecanoate reduced sperm counts following 12 weeks of therapy in only one of seven men, and his sperm counts bounced back to normal quickly following the cessation of the oral testosterone. ${ }^{10}$

Another study actually showed that oral testosterone undecanoate might be a treatment for idiopathic male infertility. ${ }^{11}$

While we do not recommend treating infertile men with oral testosterone undecanoate, these studies do indicate that it is highly unlikely that the short course of oral testosterone used by this man lead to prolonged azoospermia.

To our knowledge, this is the first documented case of semen parameters improving with the use of CC while the man remains on finasteride. This report is of interest, as low-dose finasteride is a commonly used medication to prevent hair loss in reproductive-aged men. While we would still suggest discontinuing the finasteride to improve fertility, this report suggests that there may be the possibility of improving semen parameters through the addition of CC, even if the patient continues to use finasteride to treat androgenic alopecia.
Competing interests: Dr. Jarvi has been an advisor for Eli Lilly and has received research grants from/participated in clinical studies for Allergan. The remaining authors report no competing personal or financial interests.

This paper has been peer-reviewed.

\section{References}

1. Stoner E. The clinical development of a 5 alpha-reductase inhibitor, finasteride. J Steroid Biochem Mol Biol 1990;37:375-8. https://doi.org/10.1016/0960-0760(90)90487-6

2. Amory JK, Wang C, Swerdloff RS, et al. The effect of 5alpha-reductase inhibition with dutasteride and finasteride on semen parameters and serum hormones in healthy men. J Clin Endocrinol Metab 2007;92:1659-65. https://doi.org/10.1210/ic.2006-2203

3. Samplaski MK, Lo K, Grober E, et al. Finasteride use in the male infertility population: Effects on semen and hormone parameters. Fertil Steril 2013;100:1542-6. https://doi.org/10.1016/iffertnstert.2013.07.2000

4. Tu HY, Zini A. Finasteride-induced secondary infertility associated with sperm DNA damage. Fertil Steril 2011;95:2125.e13-4.

5. Liu KE, Binsaleh S, Lo KC, et al. Propecia-induced spermatogenic failure: A report of two cases. Fertil Steril 2008;90:849 e17-9.

6. Chiba K, Yamaguchi K, Li F, et al. Finasteride-associated male infertility. Fertil Steril 2011;95:1786.e9-11.

7. Chehab M, Madala A, Trussell JC. On-label and off-label drugs used in the treatment of male infertility. Fertil Steril 2015;103:595-604. https://doi.org/10.1016/iffertnstert.2014.12.122

8. Ronnberg $L$. The effect of clomiphene citrate on different sperm parameters and serum hormone levels in preselected infertile men: a controlled double-blind cross-over study. Int I Androl 1980;3:479-86. https://doi.org/10.1111/i.1365-2605.1980.tb00136.x

9. Wang C, Chan CW, Wong KK, et al. Comparison of the effectiveness of placebo, clomiphene citrate, mesterolone, pentoxifylline, and testosterone rebound therapy for the treatment of idiopathic oligospermia. Fertil Steril 1983;40:358-65. hitps://doi.org/10.1016/S0015-0282(16)47300-0

10. Nieschlag $E$, Hoogen $H$, Bolk M, et al. Clinical trial with testosterone undecanoate for male fertility control. Contraception 1978;18:607-14. https://doi.org/10.1016/0010-7824(78)90045-8

11. Gregoriou 0 , Papadias C, Gargaropoulos A, et al. Treatment of idiopathic infertility with testosterone undecanoate. A double-blind study. Clin Exp Obstet Gynecol 1993;20:9-12.

Correspondence: Dr. Keith Allen Jarvi, Division of Urology, Department of Surgery, Mount Sinai Hospital, University of Toronto, Toronto, ON, Canada; kjarvi@mtsinai.on.ca 